Je n'ai jamais vu de Thanaos Tages Linné provenant authentiquement d'Algérie, pas plus que Adopaea Thaumas Hufn. et Augiades sylvanus Esper.

Dans le volume IV des Études de Lépidoptérologie comparée, j’ai eu tort de dire que Augiades comma Linné se rencontrait en Algérie. Je possédais alors une seule $\subsetneq$, assez mauvaise, de Benuncas que j'avais rapportée à tort à comma. Ce ne fut qu'après être entré en possession du matériel considérable dont je suis redevable à M. HaroldPowell, que j'ai distingué de comma l'espèce algérienne Benuncas, si curieusement analogue à l'américaine Uncas.

La figure de Benuncas ${ }^{\star}$ et $\subsetneq$, en dessus et en dessous, sera publiée dans le volume VIII des Études de Lépidoptérologie comparée, en mème temps que la représentation de plusieurs espèces nouvelles de Lépidoptères-Hétérocères algériens.

\title{
Deux variétés nouvelles de Chrysopides [NEvR.]
}

par J. LACROIX.

Parmi mes dernières captures de Névroptères, je signalerai deux formes nouvelles de Chrysopides de la faune française.

a) Chrysopa flavifrons Brau. var. difformis, n. var. Venulae gradatae 2/7 aut 2/6 omnino nigrae in quatuor alis (fig. 1).

Cette variété présente une ligne arquée rougeàtre en dedans des yeux, sous chaque antenne; sur la joue, un assez gros point brun rougeâtre légèrement entouré de cette dernière nuance. Sur les bords du clypeus, une strie très fine de mème couleur. Le prothorax a, sur la partie dorsale, deux lignes plus ou moins distinctes, noires, disposées en zigzag. Quelques nervules de la base aux deux ailes noires en entier; les costales, à l'aile supérieure, longuement noires aux deux extrémités, surtout celles de la base; les mèmes nervules, à l'aile inférieure, entièrement noires. Nervules radiales à l'aile supérieure, longuement noires aux deux extrémités; celles placées plıs près de l'apex de l'aile ne sont marquées qu'à une seule extrémité (celle qui tombe sur la nervure radiale) ou peuvent mème être totalement vertes. 
Par les points et les taches de la face, la variété difformis s'éloigne du type pour se rapprocher surtout de la variété riparia Ed. Pictet et de la variété Meyeri Ed. Pictet. Chez difformis toute-

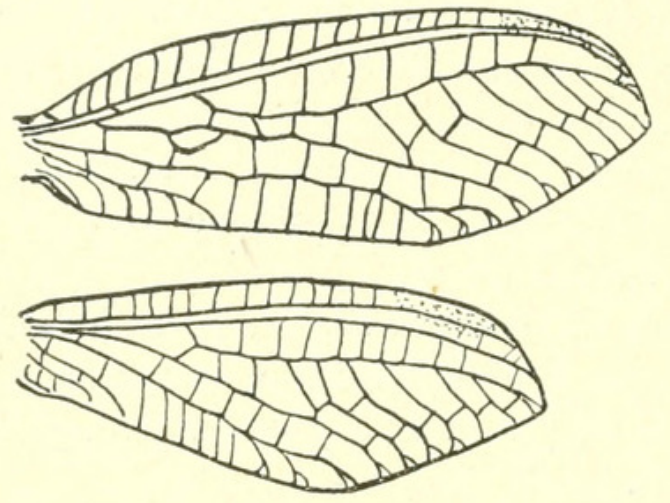

Fig. 1. - Chrysopa flavifrons B rau var. difformis $\mathrm{L}$ a croix, ailes droites. fois, le point de la joue n'est pas d'un noir pur. Par le petit nombre de ses nervules en gradins, elle a quelque analogie avec la variété geniculata Ed. Pictet qui a $3 / 4$. Elle en diffère par les deux lignes toujours suffisamment marquées du prothorax, par le point assez gros de la joue et enfin par le nombre des nervules gradiformes.

La varièté difformis mesure 23 à $24 \mathrm{~mm}$. d'envergure.

Chrysopa flavifrons $\mathrm{Brau}$. est une espèce très protéiforme. La variété que je décris aujourd'hui, sans être la plus petite, présente néanmoins une diminution manifeste des nervules gradiformes sur les deux ailes. Sous ce rapport elle est, actuellement, la plus dégradée du groupe.

Forêt de Mervent (Vendée) et Bessines près Niort (Deux-Sèvres).

b) Chrysopa ventralis Curt. var. Jodoti, n. var. - Vertice duobus punctis fuscis insignito. Primo articulo antennarum supra ad apicem puncto fusco notato.

J'ai pu capturer, le $\mathbf{2 4}$ juin 1912, dans la forêt de l'Hermitain (DeuxSèvres), un certain nombre de Chrysopa ventralis Curt., dont plusieurs individus se détachaient très nettement du type.

Le prothorax a deux points dorsaux suivis d'une trainée moins franchement noire qui va jusqu'au mésothorax. Les deux points du mésothorax assez gros. A l'aile supérieure, les nervules costales de la base noires en entier, les dernières près du ptérostigma moins ou incomplètement marquées. A l'aile inférieure, presque toutes les costales noires. Les nervules radiales, dans les deux ailes, sont presque toutes noires aux deux extrémités.

Cellules procubitales type de cette même couleur dans sa moitié terminale. Nervules en gradins $7 / 9$ ou $7 / 8$ aux ailes supérieures et $6 / 8$ ou $\breve{5} / 7$ aux ailes inférieures. Elles sont noires entièrement.

Cette variété est dédiée à M. Paul JoDot, attaché au Muséum national d'Histoire naturelle, en souvenir de notre amitié déjà ancienne. 
La variété Jodoti, par le point du premier article des antennes, a quelque analogie avec la variété pullata $\mathrm{Nav}$, mais elle s'en sépare très nettement par les marques du vertex et aussi par le point interantennal qui est simple (trilobé inférieurement dans pullata).

Notons que la présence de points sur le vertex et sur le dessus du premier article des antennes est un fait assez fréquent chez les Chrysopides de la section des Maculatae.

\section{Bulletin bibliographique.}

Andrieu et A. Vulletet : Notes sur le Sphenoptera gossypii Cotes, Buprestide nuisible au cotonnier au Soudan francais. (Insecta) 1912;

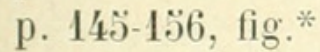

Bergroth : Additions to C. Stål's "Hemiptera fabriciana ». (Entom. Meddel.) 1912; p. 33̈9-363.*

ID. : An American genus of Henestarinae. (Hem. Myodochidae) (Ann. Soc. Ent. Belg.) 1912; p. 147-149.*

ID. : New or little known Ethiopian Hemiptera. (Ann. and Mag. of Nat. Hist.) 1912; p. 191-203. *

ID. : Notes on Coreidae and Neididae (Hém.). (Ann. Soc. Ent. Belg.) 1912; p. $76-93$.*

Borcea : Crustacés phyllopodes de Roumanie (fig.). Ann. sc. Univ. de Jassy) 1912; p. 187-208.*

ID. : Déformations provoquées par Exobasidium rhododendri Cam. sur Rhododendron myrtifolium (fig.) (Ann. sc. Univ. de Jassy) 1912; p. $209-210 . *$

ID. : Zoocecidii din Romania (Academia Rominâ) 1912; in-4 $4^{\circ} 129$ pp. $19 \mathrm{pl}^{*}$

Bugnion : Le bruissement des Termites. (Bull. Soc. Ent. Suisse) 1912; $11 \mathrm{p.}^{*}$

ID. : Eutermes lacustris nov. sp. de Ceylan (2 pl.) (Rev. Suisse zool.) 1912; p. 487-ว04.*

ID. : Observations sur les Termites. Différenciation des castes (fig.). Comptes rendu Soc. Biologie) 1912; 4 pp.* 


\section{$2 \mathrm{BHL}$ Biodiversity Heritage Library}

Lacroix, J L. 1912. "Deux variétés nouvelles de Chrysopides [Nevr.]." Bulletin de la Société entomologique de France 1912, 351-353.

View This Item Online: https://www.biodiversitylibrary.org/item/36402

Permalink: https://www.biodiversitylibrary.org/partpdf/40316

\section{Holding Institution}

Smithsonian Libraries

\section{Sponsored by}

Smithsonian

\section{Copyright \& Reuse}

Copyright Status: NOT_IN_COPYRIGHT

This document was created from content at the Biodiversity Heritage Library, the world's largest open access digital library for biodiversity literature and archives. Visit BHL at https://www.biodiversitylibrary.org. 